International Journal of Modern Physics: Conference Series

(C) The Authors

\title{
On equations of motion in twist-four evolution
}

\author{
Yao Ji, A.V. Belitsky \\ Department of Physics, Arizona State University, Tempe, AZ 85287-1504, USA
}

\begin{abstract}
Explicit diagrammatic calculation of evolution equations for high-twist correlation functions is a challenge already at one-loop order in QCD coupling. The main complication being quite involved mixing pattern of the so-called non-quasipartonic operators. Recently, this task was completed in the literature for twist-four nonsinglet sector. Presently, we elaborate on a particular component of renormalization corresponding to the mixing of gauge-invariant operators with QCD equations of motion. These provide an intrinsic contribution to evolution equations yielding total result in agreement with earlier computations that bypassed explicit analysis of Feynman graphs.
\end{abstract}

Keywords: QCD, twist-four operators, renormalization, QCD equations of motion.

PACS numbers: 12.38.Cy, 12.39.St, 25.30.Fj

\section{Introduction}

Construction of renormalization group evolution equations for twist-four operators represents quite a formidable task even at leading order of QCD perturbative series. The main obstacle being, that compared to, say, the twist-three sector ${ }^{1]}$ where the entire operator basis can be built from the so-called quasipartonic operators ${ }^{2}{ }^{2}$ it is no longer the case for higher twists. The reason for this is that the QCD equations of motions and relations based on Lorentz symmetry ${ }^{1}$ are not sufficient to eliminate non-quasipartonic operators from the set. As it was established more than three decades ago, the quasipartonic operators are distinguished by their property that one can use for them on-shell elementary states in evaluation of corresponding evolution kernels and the resulting renormalization group operator takes a pair-wise form at one-loop, conserving the number of fields in correlation functions in question.2] This virtue ceases to be the case when one goes outside of the quasipartonic states. Here off-shell nature of the particles involved mixes states with different number of fields. However, since the quasipartonic operators possess the maximal number of fields per given twist, the mixing is unidirectional, only transition of non-quasipartonic operators with smaller number of fields is possible into operators with the same number of fields or larger. For instance, for twist-four operators a

This is an Open Access article published by World Scientific Publishing Company. It is distributed under the terms of the Creative Commons Attribution 3.0 (CC-BY) License. Further distribution of this work is permitted, provided the original work is properly cited. 
generic form of the evolution equations admits the form

$$
\frac{d}{d \ln \mu}\left(\begin{array}{c}
O_{2} \\
O_{3} \\
O_{4}
\end{array}\right)=\left(\begin{array}{ccc}
K_{2 \rightarrow 2} & K_{2 \rightarrow 3} & K_{2 \rightarrow 4} \\
0 & K_{3 \rightarrow 3} & K_{3 \rightarrow 4} \\
0 & 0 & K_{4 \rightarrow 4}
\end{array}\right)\left(\begin{array}{c}
O_{2} \\
O_{3} \\
O_{4}
\end{array}\right) .
$$

Here $O_{N}$ is an operator with $N$ elementary QCD fields, with $N=4$ standing for the quasipartionic operator in conventional classification. As we alluded to above, the mixing matrix takes a triangular form such that quasipartonic operators $\mathrm{O}_{4}$ form an autonomous sector. The latter was studied in full detail decades ago. $2[3][4][6$ Thus the goal of other studies was to fill the gap by analyzing $K_{2 \rightarrow n}$ and $K_{3 \rightarrow n}$ transitions.

Over the years, only partial results were collected in the literature.7/8 ${ }^{7}$ o alleviate the complexity of brute-force perturbative calculations, a framework ${ }^{9}$ that heavily employs consequences of conformal boosts and Poincaré transformations the transverse plane has been designed and used to find coordinate-space evolution kernels for twist-four operator basis. Notice that a proper choice allowed one to eliminate $2 \rightarrow n$ transitions altogether thus further simplifying the computation. In a parallel development, a direct diagrammatic analysis was performed in the momentum-fraction space $\frac{10}{10}$ The latter is a Fourier transform of the aforementioned coordinate representation and is used in implementation of evolution in data analyses.

The use of momentum representation and light-cone gauge $A^{+}=0$ in the aforementioned calculation allowed us to reduce one-loop computations to a set of algebraic, though, quite involved manipulations. Staying away from kinematical boundaries not a single loop integral had to be performed. To confirm our calculation a Fourier transformation was performed and agreement was found with the light-ray calculation.$^{9}$ Though, the analysis of Ref ${ }^{[0}$ was quite detailed, in the present note we will put an emphasis on a most subtle aspect of that calculation, namely, the use of QCD equations of motion for transitions preserving or increasing the number of fields in operators in question. In this note, we will limit ourselves to a few explicit examples pertinent to the use of quark and gluon equations of motion in construction of twist-four evolution equations.

\section{Quark equation of motion}

To start with, let us consider the twist-three sub-block $\psi_{-}^{i}\left(x_{1}\right) \psi_{+}^{j}\left(x_{2}\right)$ built from $\mathrm{bad} / \operatorname{good}$ components $\psi_{\mp}^{i}$ of the quark field $\psi^{i}=\psi_{+}^{i}+\psi_{-}^{i}$ of a twist-four operator and unravel the contribution from the quark equation of motion in the transition channel involving the quasipartonic operator $\psi_{-}^{i}\left(x_{1}\right) \psi_{+}^{j}\left(x_{2}\right) \rightarrow$ $\psi_{+}^{i}\left(y_{1}\right) \psi^{j}\left(y_{2}\right) \bar{f}_{++}^{d}\left(y_{3}\right)$. This is shown at the diagrammatic level in Fig. 1 (a) and 


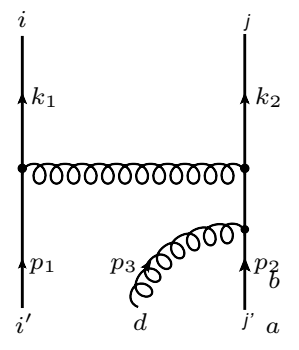

(a)

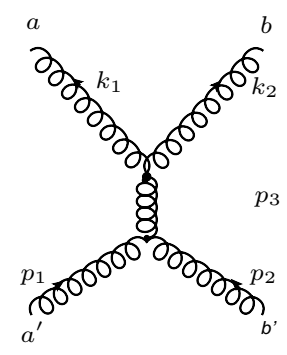

(b)

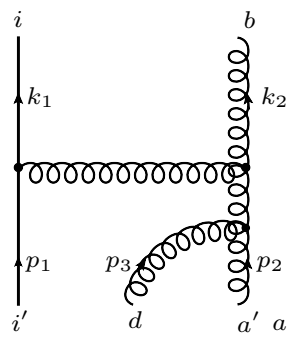

(c)

Fig. 1. Diagrams corresponding to the use of quark and gluon equations of motion in (a) and $(\mathrm{b}, \mathrm{c})$, respectively.

reads in terms of momentum integrals

$$
\begin{aligned}
& \mathcal{O}_{i j}\left(x_{1}, x_{2}\right)=\int \prod_{i=1}^{3} \frac{d^{4} p_{i}}{(2 \pi)^{4}} \delta\left(p_{i}^{+}-y_{i}\right) \prod_{j=1}^{2} \frac{d^{4} k_{j}}{(2 \pi)^{4}} \delta\left(k_{j}^{+}-x_{j}\right) \bar{\psi}_{i^{\prime}}\left(p_{1}\right) i \mathcal{V}_{\mu}^{a}\left(p_{1},-k_{1},-k_{3}\right) \\
& \times i \mathcal{P}\left(k_{1}\right) \Gamma^{-+} i \mathcal{P}\left(-k_{2}\right) i \mathcal{V}_{\nu}^{b}\left(p_{2}+p_{3},-k_{2}, k_{3}\right) i \mathcal{P}\left(-p_{2}-p_{3}\right) i \mathcal{V}_{\rho}^{c}\left(-p_{2}-p_{3}, p_{2}, p_{3}\right) \psi_{j^{\prime}}\left(p_{2}\right) \\
& \quad \times A_{\perp}^{d \rho}\left(p_{3}\right)(-i) \Delta_{\mu \nu}^{a b}\left(k_{3}\right) .
\end{aligned}
$$

Here the operator $\Gamma^{-+}$-matrix, projecting out on appropriate components of fourdimensional Dirac spinors, and the quark-gluon interaction vertex are, respectively,

$$
\Gamma^{-+}=\frac{1}{2 \sqrt{2}} \gamma^{-} \gamma^{+}\left(1+\gamma^{5}\right), \quad \mathcal{V}_{\mu}^{a}\left(k_{1}, k_{2}, k_{3}\right)=g t^{a} \gamma^{\mu}(2 \pi)^{4} \delta^{4}\left(k_{1}+k_{2}+k_{3}\right)
$$

while the quark and light-cone-gauge gluon propagator read

$$
\mathcal{P}(k)=\frac{\not k}{k^{2}}, \quad \Delta_{\mu \nu}^{a b}(k)=\frac{d_{\mu \nu}(k)}{k^{2}}, \quad d_{\mu \nu}(k)=g_{\mu \nu}-\frac{k_{\mu} n_{\nu}+k^{\nu} n_{\mu}}{k_{+}} .
$$

Denoting the integrand in Eq. (2) by $\mathcal{N} / \mathcal{D}$, we can work out the denominator $\mathcal{D}$ originated from the propagators as $\mathcal{D}=k_{1}^{2}\left(p_{1}-k\right)^{2}\left(p_{1}+p_{2}+p_{3}-k_{1}\right)^{2}\left(p_{2}+\right.$ $\left.p_{3}\right)^{2}$. Notice that since all field lines of the quasipartonic quark-gluon operator $\psi_{+}^{i}\left(y_{1}\right) \psi^{j}\left(y_{2}\right) \bar{f}_{++}^{d}\left(y_{3}\right)$ are on-shell, one immediately encounters a problem. Namely, the external legs possess collinear momenta $p_{i}=\left(p_{i}^{+}, 0, \mathbf{0}_{\perp}\right)$ with $p_{i}^{+}=y_{i}$ and thus the propagator $\left(p_{2}+p_{3}\right)^{2}$ diverges. To alleviate the problem, one has to properly regularize this. One option is to give a non-vanishing minus component $p_{i}^{-}$to particles' momenta. This was done in the past in Refs. 1 . Presently, we follow a different route and use instead the transverse momentum as a regulator. Choosing the loop momentum as $k=k_{1}$, we define $p \equiv p_{1}+p_{2}+p_{3}, q \equiv p_{2}+p_{3}$, giving the latter a non-vanishing transverse component while keeping $q^{-}=p_{2}^{-}+p_{3}^{-}=0$. This yields a regularized intermediate propagator $q^{2}=2 q^{+} q^{-}-\boldsymbol{q}_{\perp}^{2}=-\boldsymbol{q}_{\perp}^{2}$. Expanding the denominator $\mathcal{D}$ in the inverse powers of the loop's transverse momentum $\boldsymbol{k}_{\perp}$, we 
find to the lowest few orders

$$
\begin{aligned}
\frac{1}{\mathcal{D}} & =\frac{1}{\boldsymbol{k}_{\perp}^{6} \boldsymbol{q}_{\perp}^{2}} \frac{1}{\left[k^{+} \beta-1\right]\left[\left(k^{+}-p_{1}^{+}\right) \beta-1\right]\left[\left(k^{+}-p^{+}\right) \beta-1\right]} \\
& \times\left[1-\frac{2 \boldsymbol{p}_{1, \perp} \cdot \boldsymbol{k}_{\perp}}{\boldsymbol{k}_{\perp}^{2}\left[\left(k^{+}-p_{1}^{+}\right) \beta-1\right]}-\frac{2 \boldsymbol{p}_{1, \perp} \cdot \boldsymbol{k}_{\perp}}{\boldsymbol{k}_{\perp}^{2}\left[\left(k^{+}-p^{+}\right) \beta-1\right]}\right]+O\left(1 / \boldsymbol{k}_{\perp}^{8}\right) .
\end{aligned}
$$

Here we kept only terms that induce logarithmic dependence on the ultraviolet cutoff $\mu$ in transverse momentum integrals. These are the renormalization-group logs that we are resumming. In the above equation, we introduced the $\beta$-variable as a rescaled minus component of the loop momentum $\beta=2 k^{-} / \boldsymbol{k}_{\perp}^{2}$. The Dirac algebra in the numerator

$$
\mathcal{N}=-i g^{3}\left(t^{a}\right)_{i i^{\prime}}\left(t^{a} t^{d}\right)_{j j^{\prime}} \bar{\psi}_{i^{\prime}}\left(p_{1}\right)\left[\gamma^{\mu} \not k \Gamma^{-+}(\not k-\not p) \gamma^{\nu} \not 1 A_{\perp}^{d}\right] \psi_{j^{\prime}}\left(p_{2}\right) d_{\mu \nu}\left(k-p_{1}\right),
$$

can be easily performed by means of Sudakov decomposition of all momenta, loop and external. Working this out and performing momentum integrations, with $k^{+}$ momentum simply eliminated by means of the delta-function constraints, $k^{-}$momentum (a.k.a. $\beta$ ) generating generalized step-functions

$$
\vartheta_{\alpha_{1}, \ldots, \alpha_{n}}^{k}\left(x_{1}, \ldots, x_{n}\right)=\int_{-\infty}^{\infty} \frac{d \beta}{2 \pi i} \beta^{k} \prod_{\ell=1}^{n}\left(x_{\ell} \beta-1+i 0\right)^{-\alpha_{\ell}},
$$

and, finally, the $\boldsymbol{k}_{\perp}$ one yielding $\ln \mu$ dependence, one immediately arrives at the result

$$
\begin{aligned}
\mathcal{O}_{i j}\left(x_{1}, x_{2}\right) & =-\frac{g^{3} \ln \mu}{16 \sqrt{2} \pi^{2} \boldsymbol{q}_{\perp}^{2}} t_{i i^{\prime}}^{a}\left(t^{a} t^{d}\right)_{j j^{\prime}} \\
& \times \int d \mathcal{M}_{3} \bar{\psi}_{i^{\prime}}\left(p_{1}\right)\left[\frac{\boldsymbol{q}_{\perp}^{2} \gamma^{+} \mathscr{A}_{\perp}^{d}\left(p_{3}\right) \vartheta_{111}^{0}\left(x_{1}, x_{1}-y_{1},-x_{2}\right)}{x_{1}-y_{1}}+\ldots\right] \psi_{j^{\prime}}\left(p_{2}\right),
\end{aligned}
$$

where here and below we used the three-particle measure

$$
d \mathcal{M}_{3} \equiv \prod_{i=1}^{3} \frac{d p_{i}^{-} d^{2} \boldsymbol{p}_{i, \perp}}{(2 \pi)^{4}} \delta\left(\sum_{i=1}^{3} y_{i}-\sum_{j=1}^{2} x_{j}\right) .
$$

Since Fig. 1 (a) corresponds to the quark equation of motion $\not p \psi(p)=$ $-g \int d^{4} p^{\prime} A\left(p^{\prime}\right) \psi\left(p-p^{\prime}\right)$ and $q q=q^{2}=-\boldsymbol{q}_{\perp}^{2}$, we only need to keep terms proportional to $\boldsymbol{q}_{\perp}^{2}$ thus neglecting everything else denoted by ellipses in the right-hand side of the above equation. Effectively, the use of the quark equation of motion can be understood as a contraction of the fermion propagator $i \mathcal{P}(-q)$ into a point. Thus, we get the final addendum to the rest of the evolution kernel

$$
\begin{aligned}
\mathcal{O}_{i j}\left(x_{1}, x_{2}\right) & =\frac{\sqrt{2} g^{3}}{32 \pi^{2}} t_{i i^{\prime}}^{a}\left(t^{a} t^{d}\right)_{j j^{\prime}} \ln \mu \\
& \times \int d \mathcal{M}_{3} \frac{\vartheta_{111}^{0}\left(x_{1}, x_{1}-y_{1},-x_{2}\right)}{x_{1}-y_{1}} \bar{\psi}_{i^{\prime}}\left(p_{1}\right) \gamma^{+} A_{\perp}^{d}\left(p_{3}\right) \psi_{j^{\prime}}\left(p_{2}\right),
\end{aligned}
$$


where $\gamma^{+} A_{\perp}$ provides the correct Dirac structure for the channel. Only after this contribution is accounted for, we total results coincides with the one available in the literature.

\section{Gluon equation of motion}

Moving on to the gluon equations of motion, $D_{\mu} F^{\mu \nu}=g j^{\nu}$, the same story applies without significant modifications. It becomes more involved though due to Lorentz structures of contributing diagrams. Since the focus of our study was a nonsinglet sector, we are not concerned about gluon-quark transition scenarios. Therefore, we can safely set the QCD quark current to zero, $j^{\nu}=0$ and simplify the gluon equation of motion to just pure gluodynamics $D_{\mu} F^{\mu \nu}=0$. Decomposing it in terms of Sudakov components, we get

$$
\begin{aligned}
& \left(\partial^{+}\right)^{2} A^{a-}-\partial_{\top} A_{\perp}^{a}-\partial_{\perp} A_{\top}^{a}-g f^{a b c}\left(A_{\perp}^{b} A_{\top}^{c}+A_{\top}^{b} A_{\perp}^{c}\right)=0, \\
& \partial^{+}\left(F^{-\perp} \pm F^{-\top}\right)+D^{-}\left(F^{+\perp} \pm F^{+\top}\right) \\
& \quad+\frac{1}{2}\left(D^{\perp} \mp D^{\top}\right)\left[ \pm\left(F^{\perp \perp}+F^{\perp \top}\right) \mp\left(F^{\top \perp}+F^{\top \top}\right)\right]=0,
\end{aligned}
$$

in the light cone gauge $A^{+}=0$. Here, we introduced conventional notations for helicity plus/minus gluon fields

$$
A_{\perp}=A^{1}+i A^{2}, \quad A_{\top}=A^{1}-i A^{2} .
$$

The same notation will be used below for holomorphic and antiholomorphic components of any four-vector.

In practice, the use of gluonic equations of motion in Feynman graphs is employed by keeping contributions in numerators that cancel denominators of on-shell propagators, in a fashion identical to the one for quarks. Namely, giving these lines a small transverse momentum does the trick. Let us illustrate this methods with a few examples.

We start with a simple example of a two-to-two particle transition $f_{++}^{a}\left(x_{1}\right) \bar{f}_{++}^{b}\left(x_{2}\right) \rightarrow f_{++}^{a^{\prime}}\left(y_{1}\right) \bar{f}_{++}^{b^{\prime}}\left(y_{2}\right)$ that, due to helicity-conservation, possesses an nontrivial annihilation channel as shown in Fig. 1(b). This graph yields

$$
\begin{aligned}
\mathcal{O}^{a b}\left(x_{1}, x_{2}\right) & =\int \prod_{i=1}^{2} \frac{d^{4} p_{i}}{(2 \pi)^{4}} \delta\left(p_{i}^{+}-y_{i}\right) \prod_{j=1}^{2} \frac{d^{4} k_{j}}{(2 \pi)^{4}} \delta\left(k_{j}^{+}-x_{j}\right) \frac{k_{1}^{+} k_{2}^{+}}{p_{1}^{+} p_{2}^{+}} \\
& \times \Gamma^{\rho \mu \nu \sigma} \mathcal{V}_{\delta \lambda \alpha}^{b_{1} b_{2} a_{1}}\left(-k_{2}, p_{1}+p_{2},-k_{1}\right) \mathcal{V}_{\rho \sigma \tau}^{a^{\prime} b^{\prime} b_{3}}\left(p_{1}, p_{2},-p_{1}-p_{2}\right) \\
& \times(-i) \Delta_{a a_{1}}^{\mu \nu}\left(k_{1}\right)(-i) \Delta_{b b_{1}}^{\nu \delta}\left(k_{2}\right)(-i) \Delta_{b_{2} b_{3}}^{\lambda \tau}\left(p_{1}+p_{2}\right) A_{\perp}^{a^{\prime}}\left(p_{1}\right) A_{\top}^{b^{\prime}}\left(p_{2}\right),
\end{aligned}
$$

where we use for convenience a projector in terms of the Dirac matrices $\Gamma^{\rho \mu \nu \sigma}=$ $\gamma_{\perp}^{\rho} \gamma_{T}^{\mu} \gamma_{\perp} \gamma_{\perp}^{\nu} \gamma_{T}^{\sigma}$ on the transverse holomorphic and antiholomorphic components of the gluon field, and the three-gluon vertex being

$$
\begin{aligned}
\mathcal{V}_{\mu \nu \rho}^{a b c}\left(k_{1}, k_{2}, k_{3}\right) & =(2 \pi)^{4} \delta^{(4)}\left(k_{1}+k_{2}+k_{3}\right) \\
& \times g f^{a b c}\left[\left(k_{1}-k_{2}\right)_{\rho} g_{\mu \nu}+\left(k_{2}-k_{3}\right)_{\mu} g_{\nu \rho}+\left(k_{3}-k_{1}\right)_{\nu} g_{\rho \mu}\right] .
\end{aligned}
$$


We also defined the transverse four vectors of Dirac matrices $\gamma_{\perp}^{\mu}, \gamma_{\top}^{\mu}$ and their (anti)holomorphic combinations $\gamma_{\perp}$ and $\gamma_{\top}$,

$\gamma_{\perp}^{\mu}=\left(0, \gamma^{1}, \gamma^{2}, 0\right), \quad \gamma_{\top}^{\mu}=\left(0, \gamma^{1},-\gamma^{2}, 0\right), \quad \gamma_{\perp}=\gamma^{1}+i \gamma^{2}, \quad \gamma_{\top}=\gamma^{1}-i \gamma^{2}$

After some algebra and integration, we arrive at the expression

$$
\begin{aligned}
\mathcal{O}^{a b}\left(x_{1}, x_{2}\right) & =\frac{g^{2} \ln \mu}{\pi^{2}} f^{b c a} f^{a^{\prime} b^{\prime} c} \gamma_{\perp} \\
& \times \int d \mathcal{M}_{2} A_{\perp}^{a_{\perp}^{\prime}}\left(p_{1}\right) A_{\top}^{b^{\prime}}\left(p_{2}\right) \frac{x_{1} x_{2}\left(x_{1}-x_{2}\right)\left(y_{1}-y_{2}\right)\left(\vartheta\left(-x_{2}\right)-\vartheta\left(x_{1}\right)\right)}{y_{1} y_{2}\left(y_{1}+y_{2}\right)^{3}}
\end{aligned}
$$

with now two-particle measure

$$
d \mathcal{M}_{2} \equiv \prod_{i=1}^{2} \frac{d p_{i}^{-} d^{2} \boldsymbol{p}_{i, \perp}}{(2 \pi)^{4}} \delta\left(\sum_{i=1}^{2} y_{i}-\sum_{j=1}^{2} x_{j}\right)
$$

Above, $\vartheta(x)$ is the ordinary step function and $\gamma_{\perp}$ structure is maintained to the end as anticipated. The expression above is obtained through the cancellation of the denominator of the on-shell propagator $\left(p_{1}+p_{2}\right)^{2}=-\left(\boldsymbol{p}_{1}+\boldsymbol{p}_{2}\right)_{\perp}^{2}$ and ignoring all terms that fail to cancel $\left(p_{1}+p_{2}\right)^{-2}$. In fact, the terms that remove the singular dependence on "divergent" propagator $\left(p_{1}+p_{2}\right)^{-2}$ are the only term that survive to the end.

Let us now outline the strategy for a two-to-three transitions, shown in Fig. 1 (c). This graphs correspond to the a particular contribution to the transition $\frac{1}{2} D_{-+} \bar{\psi}_{+}^{i}\left(x_{1}\right) \bar{f}_{++}^{a}\left(x_{2}\right) \rightarrow \bar{\psi}_{+}^{i}\left(y_{1}\right) \bar{f}_{++}^{a}\left(y_{2}\right) \bar{f}_{++}^{d}\left(y_{3}\right)$. Using the vertex $\Gamma^{\rho \chi}=$ $\frac{\sqrt[4]{2}}{2} \gamma^{+} \gamma_{T} \gamma_{T}^{\rho} \gamma_{T}^{\chi}$ in four-dimensional notations, we can write the momentum integrals for the transition in question as

$$
\begin{aligned}
\mathcal{O}^{i a}\left(x_{1}, x_{2}\right) & =\int \prod_{i=1}^{3} \frac{d^{4} p_{i}}{(2 \pi)^{4}} \delta\left(p_{i}^{+}-y_{i}\right) \prod_{j=1}^{2} \frac{d^{4} k_{1}}{(2 \pi)^{4}} \delta\left(k_{j}^{+}-x_{j}\right) \bar{\psi}_{i^{\prime}}\left(p_{1}\right) i \mathcal{V}_{\mu}^{b_{1}}\left(p_{1},-k_{1},-k_{3}\right) \\
& \times i \mathcal{P}\left(k_{1}\right) \Gamma^{\rho \chi} k_{1 \top} A_{\perp}^{d, \alpha}\left(p_{3}\right) \mathcal{V}_{\nu \sigma \lambda}^{b_{2} b_{4} b_{3}}\left(k_{3}, p_{2}+p_{3},-k_{2}\right) \mathcal{V}_{\alpha \chi \tau}^{d a b_{5}}\left(p_{3}, p_{2},-p_{2}-p_{3}\right) \\
& \times(-i) \Delta_{b_{1} b_{2}}^{\mu \nu}\left(k_{1}\right)(-i) \Delta_{c b_{3}}^{\rho \sigma}\left(k_{2}\right)(-i) \Delta_{b_{4} b_{5}}^{\lambda \tau}\left(p_{2}+p_{3}\right) A_{\top}^{a^{\prime}}\left(p_{2}\right)
\end{aligned}
$$

where as before $k_{1 \top}=k^{1}-i k^{2}$ and $k_{1 \perp}=k^{1}+i k^{2}$. After some algebraic manipulations that cancel the on-shell propagator and straightforward integrations over the loop momentum, we end up with the following expression

$$
\begin{aligned}
\mathcal{O}^{i a}\left(x_{1}, x_{2}\right) & =-\frac{\sqrt[4]{2} i g^{3}}{8 \pi^{2}} t_{i i^{\prime}}^{b_{1}} f^{b_{1} b_{4} a} f^{d a^{\prime} b_{4}} \\
& \times \int d \mathcal{M}_{3} V\left(x_{1}, x_{2} \mid y_{1}, y_{2}, y_{3}\right) \bar{\psi}_{i^{\prime}}\left(p_{1}\right) \gamma^{+} \gamma_{\top} A_{\top}^{a^{\prime}}\left(p_{2}\right) A_{\top}^{d}\left(p_{3}\right),
\end{aligned}
$$




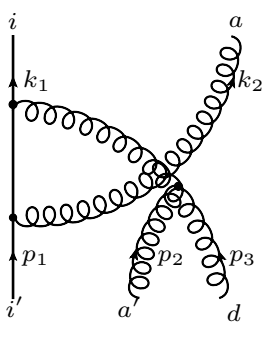

(a)

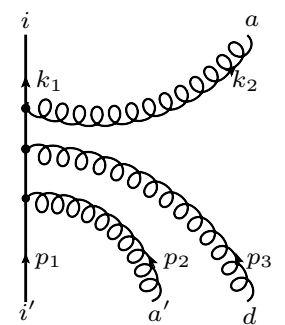

(b)

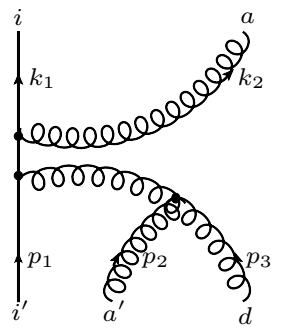

(c)

Fig. 2. In (a), the Feynman graph due to gluon equation of motion in the transition $\frac{1}{2} D_{-+} \bar{\psi}_{+}^{i}\left(x_{1}\right) f_{++}^{a}\left(x_{2}\right) \rightarrow \bar{\psi}_{+}^{i^{\prime}}\left(y_{1}\right) f_{++}^{a^{\prime}}\left(y_{2}\right) \bar{f}_{++}^{d}\left(y_{3}\right)$. In (b) and (c), diagrams corresponding to the double use of equations of motion for mixing $\frac{1}{2} D_{-+} \bar{\psi}_{+}^{i}\left(x_{1}\right) f_{++}^{a}\left(x_{2}\right) \rightarrow \bar{\psi}_{+}^{i^{\prime}}\left(y_{1}\right) f_{++}^{a^{\prime}}\left(y_{2}\right) \bar{f}_{++}^{d}\left(y_{3}\right)$ and $\frac{1}{2} D_{-+} \bar{\psi}_{+}^{i}\left(x_{1}\right) f_{++}^{a}\left(x_{2}\right) \rightarrow \bar{\psi}_{+}^{i^{\prime}}\left(y_{1}\right) f_{++}^{a^{\prime}}\left(y_{2}\right) \bar{f}_{++}^{d}\left(y_{3}\right)$, respectively.

where the transition kernel stemming from the graph 1 (c) reads

$$
\begin{aligned}
& V\left(x_{1}, x_{2} \mid y_{1}, y_{2}, y_{3}\right)=\frac{x_{2}\left[\left(y_{2}+y_{3}\right)\left(y_{1}+2\left(y_{2}+y_{3}\right)\right)-2 y_{3} x_{2}\right]}{y_{2} y_{3}\left(x_{1}-y_{1}\right)\left(y_{2}+y_{3}\right)} \vartheta_{12}^{0}\left(x_{1}-y_{1},-x_{2}\right) \\
& +\frac{2 y_{1} y_{2}\left(y_{2}+y_{3}\right)-x_{2}\left(y_{2}-y_{3}\right)\left(y_{1}+2\left(y_{2}+y_{3}\right)\right)+2 x_{2}^{2}\left(y_{2}-y_{3}\right)}{y_{2} y_{3}\left(x_{1}-y_{1}\right)\left(y_{2}+y_{3}\right)} \vartheta_{112}^{0}\left(x_{1}, x_{1}-y_{1},-x_{2}\right) \\
& +\frac{x_{1} x_{2}\left(y_{3}-y_{2}\right) \vartheta_{111}^{0}\left(x_{1}, x_{1}-y_{1},-x_{2}\right)}{y_{2} y_{3}\left(x_{1}-y_{1}\right)\left(y_{2}+y_{3}\right)}-\frac{x_{1} y_{1} x_{2} \vartheta_{112}^{1}\left(x_{1}, x_{1}-y_{1},-x_{2}\right)}{x_{1} y_{2} y_{3}-y_{1} y_{2} y_{3}}
\end{aligned}
$$

Let us conclude this section by discussing the use of gluon equations of motion when the field is emitted from an internal off-shell line. The corresponding diagram is shown in Fig. 2 (a) and reads

$$
\begin{gathered}
\mathcal{O}^{i a}\left(x_{1}, x_{2}\right)=\int \prod_{i=1}^{3} \frac{d^{4} p_{i}}{(2 \pi)^{4}} \delta\left(p_{i}^{+}-y_{i}\right) \prod_{j=1}^{2} \frac{d^{4} k_{j}}{(2 \pi)^{4}} \delta\left(k_{j}^{+}-x_{j}\right) \bar{\psi}_{i^{\prime}}\left(p_{1}\right) i \mathcal{V}_{\mu}^{b_{1}}\left(p_{1},-k_{2}, k_{2}-p_{1}\right) \\
\times i \mathcal{P}\left(k_{1}-p_{2}-p_{3}\right) i \mathcal{V}_{\rho}^{b_{2}}\left(p_{1}-k_{2}, p_{2}+p_{3},-k_{1}\right) i \mathcal{P}\left(k_{1}\right) \Gamma^{\nu \alpha} k_{1, \top} \mathcal{V}_{\chi \alpha \sigma}^{d a^{\prime} b_{3}}\left(p_{3}, p_{2},-p_{2}-p_{3}\right) \\
\quad \times(-i) \Delta_{a b_{1}}^{\mu \nu}\left(k_{2}\right)(-i) \Delta_{b_{2} b_{3}}^{\rho \sigma}\left(p_{2}+p_{3}\right) A_{\perp}^{a^{\prime}}\left(p_{2}\right) A_{\perp}^{d, \chi}\left(p_{3}\right)
\end{gathered}
$$

where $\Gamma^{\nu \alpha}=\frac{1}{2 \sqrt{2}} \gamma^{+} \gamma_{\top} \gamma_{T}^{\nu} \gamma_{T}^{\alpha}$. After dropping all terms that leave the divergent $\left(p_{2}+p_{3}\right)^{2}$ factor in the denominator intact, we find

$$
\begin{aligned}
\mathcal{O}^{i a}\left(x_{1}, x_{2}\right) & =-\frac{\sqrt{2} g^{3} \ln \mu}{4 \pi^{2}}\left(t^{a} t^{c}\right)_{i i^{\prime}} f^{d a^{\prime} c} \\
& \times \int d \mathcal{M}_{3} V\left(x_{1}, x_{2} \mid y_{1}, y_{2}, y_{3}\right) \bar{\psi}_{i^{\prime}}\left(p_{1}\right) \gamma^{+} \gamma_{\top} A_{\perp}^{a^{\prime}}\left(p_{2}\right) A_{\top}^{d}\left(p_{3}\right),
\end{aligned}
$$


with the following nontrivial evolution kernel

$$
\begin{aligned}
& V\left(x_{1}, x_{2} \mid y_{1}, y_{2}, y_{3}\right)=-\frac{\left(y_{1}^{2}-3 y_{1} x_{2}+2 x_{2}^{2}\right) \vartheta_{112}^{0}\left(x_{1}, y_{1}-x_{2},-x_{2}\right)}{y_{2} y_{3}\left(y_{2}+y_{3}\right)} \\
& +\frac{x_{1}\left(y_{1}-2 x_{2}\right) \vartheta_{22}^{0}\left(y_{1}-x_{2},-x_{2}\right)}{y_{2} y_{3}\left(y_{2}+y_{3}\right)}+\frac{\left(2 x_{2}-y_{1}\right)\left(y_{2}-y_{3}\right) \vartheta_{122}^{1}\left(x_{1}, y_{1}-x_{2},-x_{2}\right)}{y_{2} y_{3}} \\
& +\frac{\left(y_{1}\left(y_{3}-3 y_{2}\right)+2 x_{2}\left(y_{2}-y_{3}\right)\right) \vartheta_{122}^{0}\left(x_{1}, y_{1}-x_{2},-x_{2}\right)}{y_{2} y_{3}\left(y_{2}+y_{3}\right)} \\
& +\frac{4 x_{2}\left(x_{2}-y_{1}\right)\left(-y_{1}-y_{2}-y_{3}+x_{2}\right) \vartheta_{122}^{2}\left(x_{1}, y_{1}-x_{2},-x_{2}\right)}{y_{2}\left(y_{2}+y_{3}\right)}
\end{aligned}
$$

\section{Double equations of motion}

In certain mixing channels, one also has to account for graphs that involve double use of quark and/or gluon equations of motion. In these cases, the method advocated above works as well. Below, we give a couple of examples to illustrate the point. First, we will discuss the diagram, shown in Fig. 2 (b), that possesses two quark on-shell propagators. Its integral representation reads

$$
\begin{aligned}
\mathcal{O}^{i a}\left(x_{1}, x_{2}\right) & =\int \prod_{i=1}^{3} \frac{d^{4} p_{i}}{(2 \pi)^{4}} \delta\left(p_{i}^{+}-y_{i}\right) \prod_{j=1}^{2} \frac{d^{4} k_{i}}{(2 \pi)^{4}} \delta\left(k_{j}^{+}-x_{j}\right) \bar{\psi}^{i^{\prime}}\left(p_{1}\right) i \mathcal{V}_{\rho}^{d}\left(p_{1}, p_{2},-p_{1}-p_{2}\right) \\
& \times i \mathcal{P}\left(p_{1}+p_{2}\right) i \mathcal{V}_{\chi}^{a^{\prime}}\left(p_{1}+p_{2}, p_{3},-p_{1}-p_{2}-p_{3}\right) i \mathcal{P}\left(p_{1}+p_{2}+p_{3}\right) \\
& \times i \mathcal{V}_{\mu}^{b}\left(p_{1}+p_{2}+p_{3},-k_{1},-k_{2}\right) i \mathcal{P}\left(k_{1}\right) \Gamma_{\top}^{\alpha \chi}(-i) \Delta_{a b}^{\rho \sigma}\left(k_{2}\right) k_{1 \top} A_{\perp}^{a^{\prime}}\left(p_{2}\right) A_{d \perp}^{\rho}\left(p_{3}\right),
\end{aligned}
$$

where $\Gamma_{T}^{\alpha \chi}=\frac{1}{2 \sqrt{2}} \gamma^{+} \gamma_{T} \gamma_{T}^{\alpha} \gamma_{T}^{\chi}$ projects the quark-gluon operator in question. Separating the terms with cancelled denominators $\left(p_{1}+p_{2}\right)^{2}\left(p_{1}+p_{2}+p_{3}\right)^{2}$ front the rest, we have the following contribution to the transition from double quark equation of motion,

$$
\begin{aligned}
\mathcal{O}^{i a}\left(x_{1}, x_{2}\right) & =\frac{i g^{3} \ln \mu}{2 \sqrt{2} \pi^{2}}\left(t^{d} t^{a^{\prime}} t^{a}\right)_{i i^{\prime}} \\
& \times \int d \mathcal{M}_{3} \frac{x_{1} x_{2} \vartheta_{12}^{0}\left(x_{1},-x_{2}\right)}{y_{2} y_{3}\left(x_{1}+x_{2}\right)} \bar{\psi}^{i^{\prime}}\left(p_{1}\right) \gamma^{+} \gamma_{\top} A_{\perp}^{a^{\prime}}\left(p_{2}\right) A_{\top}^{d}\left(p_{3}\right) .
\end{aligned}
$$

Last but not least, let us address the case involving both quark and gluon equations of motion. A representative graph in demonstrated in Fig. 2 (c). It provided an additive contribution to the following two-to-three transition $\frac{1}{2} D_{-+} \bar{\psi}_{+}^{i}\left(x_{1}\right) f_{++}^{a}\left(x_{2}\right) \rightarrow \bar{\psi}_{+}^{i}\left(y_{1}\right) f_{++}^{a}\left(y_{2}\right) \bar{f}_{++}^{d}\left(y_{3}\right)$ and gives

$$
\begin{aligned}
& \mathcal{O}^{i a}\left(x_{1}, x_{2}\right)=\int \prod_{i=1}^{3} \frac{d^{4} p_{i}}{(2 \pi)^{4}} \delta\left(p_{i}^{+}-y_{i}\right) \prod_{j=1}^{2} \frac{d^{4} k_{j}}{(2 \pi)^{4}} \delta\left(k_{j}^{+}-x_{j}\right) k_{1, \top} A_{\perp}^{a^{\prime} \rho}\left(p_{2}\right) A_{\perp}^{d \chi}\left(p_{3}\right) \\
& \times \bar{\psi}\left(p_{1}\right) i \mathcal{V}_{\lambda}^{b}\left(p_{1}, p_{2}+p_{3},-p_{1}-p_{2}-p_{3}\right) i \mathcal{P}\left(p_{1}+p_{2}+p_{3}\right) i \mathcal{V}_{\sigma}^{c}\left(-k_{1},-k_{2}, p_{1}+p_{2}+p_{3}\right) \\
& \times i \mathcal{P}\left(k_{1}\right) \Gamma_{\top} \mathcal{V}_{\tau \chi \rho}^{b^{\prime} d a^{\prime}}\left(-p_{2}-p_{3}, p_{3}, p_{2}\right)(-i) \Delta_{a c}^{\alpha \sigma}\left(k_{2}\right)(-i) \Delta_{b b^{\prime}}^{\lambda \tau}\left(p_{2}+p_{3}\right),
\end{aligned}
$$


with $\Gamma_{\top}=\frac{1}{2 \sqrt{2}} \gamma^{+} \gamma_{\top}$. Projecting on the channel in question, taking care of the symmetrization by swapping $A_{\perp}^{a^{\prime} \rho} \leftrightarrow A_{\perp}^{d \chi}$ and finally dropping terms with uncancelled denominator $\left(p_{2}+p_{3}\right)^{2}\left(p_{1}+p_{2}+p_{3}\right)^{2}$, we get

$$
\begin{aligned}
\mathcal{O}^{i a}\left(x_{1}, x_{2}\right) & =-\frac{g^{3} \ln \mu}{8 \sqrt{2} \pi^{2}}\left(t^{b} t^{a}\right)_{i i^{\prime}} f^{b a^{\prime} d} \\
& \times \int d \mathcal{M}_{3} \frac{x_{1} x_{2}\left(y_{2}-y_{3}\right) \vartheta_{12}^{0}\left(x_{1},-x_{2}\right)}{\left(x_{1}+x_{2}\right) y_{2} y_{3}\left(y_{2}+y_{3}\right)} \bar{\psi}^{i^{\prime}}\left(p_{1}\right) \gamma^{+} \gamma_{\top} A_{\perp}^{a^{\prime}}\left(p_{2}\right) A_{\top}^{d}\left(p_{3}\right) .
\end{aligned}
$$

\section{Conclusions}

In this note, we provided a detailed discussion of a formalism that properly incorporates graphs induced by quark and gluon equations of motion in the renormalization group evolution of twist-four operators. Neglecting these would yield an incomplete answer for corresponding evolution equations. The method uses regularization of external off-shell lines by giving them a nonvanishing transverse momentum. This techniques is a bit more advantageous compared to endowing the lines with minus momentum since the former does not admix momentum fractions in the game. When added to the rest of contributions from Feynman graphs, the complete results was found in agreement ${ }^{10}$ with recent analysis of twist-four evolution kernels by means of a different technique. ${ }^{9}$ Our formalism is not tailored particularly to the twist-four case and thus can be easily generalized to even higher twists once a complete basis of operators is properly chosen.

\section{Acknowledgments}

This work was supported by the U.S. National Science Foundation under the grant PHY-1068286.

\section{References}

1. A.P. Bukhvostov, E.A. Kuraev, L.N. Lipatov, "Evolution equations for higher twist operators," Sov. J. Nucl. Phys. 38 (1983) 263; "Deep inelastic scattering on a polarized target in Abelian gauge theory," Sov. J. Nucl. Phys. 39 (1984) 121; "Deep inelastic electron scattering by a polarized target in Quantum Chromodynamics," Sov. Phys. JETP 60 (1984) 22

2. A.P. Bukhvostov, G.V. Frolov, L.N. Lipatov, E.A. Kuraev, "Evolution equations for quasi-partonic operators," Nucl. Phys. B 258 (1985) 601.

3. A.P. Bukhvostov, G.V. Frolov , "Anomalous dimensions of quasipartonics twist-four operators," Yad. Fiz. 45 (1987) 1136.

4. A. Ali, V.M. Braun, G. Hiller, "Asymptotic solutions of the evolution equation for the polarized nucleon structure function $g_{2}\left(x, Q^{2}\right)$," Phys. Lett. B 266 (1991) 117; J. Kodaira, Y. Yasui, K. Tanaka, T. Uematsu, "QCD corrections to the nucleon's spin structure function $g_{2}\left(x, Q^{2}\right)$," Phys. Lett. B 387 (1996) 855 [hep-ph/9603377]; B. Geyer, D. Müller, D. Robaschik, "Evolution kernels of twist-three light ray operators in polarized deep inelastic scattering," Nucl. Phys. Proc. Suppl. 51C (1996) 
106 [hep-ph/9606320];

Y. Koike, K. Tanaka, " $Q 2$ evolution of nucleon's chiral odd twist-three structure function: $h_{L}\left(x, Q^{2}\right)$," Phys. Rev. D 51 (1995) 6125 [hep-ph/9412310];

Y. Koike, N. Nishiyama, " $Q 2$ evolution of chiral odd twist-three distribution $e\left(x, Q^{2}\right), "$ Phys. Rev. D 55 (1997) 3068 [hep-ph/9609207];

I.I. Balitsky, V.M. Braun, Y. Koike, K. Tanaka, " $Q^{2}$ evolution of chiral odd twistthree distributions $h_{L}\left(x, Q^{2}\right)$ and $e\left(x, Q^{2}\right)$ in the large- $N_{c}$ limit," Phys. Rev. Lett. 77 (1996) 3078 [hep-ph/9605439];

D. Müller, "Calculation of higher twist evolution kernels for polarized deep inelastic scattering," Phys. Lett. B 407 (1997) 314 [hep-ph/9701338];

A.V. Belitsky, "Leading order analysis of the twist-three space-like and time-like cut vertices in QCD," hep-ph/9703432.

A.V. Belitsky, D. Müller, "Scale dependence of the chiral odd twist-three distributions $h_{L}(x)$ and $e(x)$," Nucl. Phys. B 503 (1997) 279 [hep-ph/9702354];

5. V.M. Braun, S.E. Derkachov, A.N. Manashov, "Integrability of three particle evolution equations in QCD," Phys. Rev. Lett. 81 (1998) 2020 [hep-ph/9805225];

V.M. Braun, S.E. Derkachov, G.P. Korchemsky, A.N. Manashov, "Baryon distribution amplitudes in QCD," Nucl. Phys. B 553 (1999) 355 [hep-ph/9902375];

S.E. Derkachov, G.P. Korchemsky, A.N. Manashov, "Evolution equations for quark gluon distributions in multicolor QCD and open spin chains," Nucl. Phys. B 566 (2000) 203 [hep-ph/9909539];

V.M. Braun, G.P. Korchemsky, A.N. Manashov, "Evolution equation for the structure function $g_{2}\left(x, Q^{2}\right)$," Nucl. Phys. B 603, 69 (2001) [hep-ph/0102313].

6. A.V. Belitsky, "Fine structure of spectrum of twist-three operators in QCD," Phys. Lett. B 453 (1999) 59 [hep-ph/9902361]; "Integrability and WKB solution of twistthree evolution equations," Nucl. Phys. B 558 (1999) 259 [hep-ph/9903512]; "Renormalization of twist-three operators and integrable lattice models," Nucl. Phys. B 574 (2000) 407 [hep-ph/9907420].

7. E.V. Shuryak, A.I. Vainshtein, "Theory of power corrections to deep inelastic scattering in quantum chromodynamics. 1. $Q^{2}$ effects," Nucl. Phys. B 199 (1982) 451; "Theory of power corrections to deep inelastic scattering in quantum chromodynamics. 2. $Q^{4}$ effects: polarized target," Nucl. Phys. B 201 (1982) 141;

R.L. Jaffe, M. Soldate, "Twist four in electroproduction: canonical operators and coefficient functions," Phys. Rev. D 26 (1982) 49;

R.K. Ellis, W. Furmanski, R. Petronzio, "Power corrections to the parton model in QCD," Nucl. Phys. B 207 (1982) 1; "Unraveling higher twists," Nucl. Phys. B 212 (1983) 29;

I.I. Balitsky, V.M. Braun, "Evolution equations for QCD string operators," Nucl. Phys. B 311 (1989) 541;

J.-W. Qiu, "Twist four contributions to the parton structure functions," Phys. Rev. D 42 (1990) 30.

8. S.A. Gottlieb, "Contribution of twist four operators to deep inelastic scattering," Nucl. Phys. B 139 (1978) 125;

S. Wada, "Analysis of twist four two quark process by the renormalization mixing," Nucl. Phys. B 202 (1982) 201;

M. Okawa, "Higher twist effects in asymptotically free gauge theories: the anomalous dimensions of four quark operators," Nucl. Phys. B 172 (1980) 481; "Higher twist effects in asymptotically free gauge theories," Nucl. Phys. B 187 (1981) 71;

A.Yu. Morozov, "Calculation Of One Loop Anomalous Dimensions By Means Of The Background Field Method," ITEP-190-1983; ITEP-191-1983; "Matrix of mixing of 
scalar and vector mesons of dimension $d \leq 8$ in QCD," Sov. J. Nucl. Phys. 40 (1984) 505 ;

M.J. Glatzmaier, S. Mantry, M.J. Ramsey-Musolf, "Higher twist in electroproduction: Flavor nonsinglet QCD evolution," Phys. Rev. C 88 (2013) 2, 025202 [arXiv:1208.2998 [hep-ph]].

9. V.M. Braun, A.N. Manashov, J. Rohrwild, "Renormalization of twist-four operators in QCD," Nucl. Phys. B 826 (2010) 235 [arXiv:0908.1684 [hep-ph]];

V.M. Braun, A.N. Manashov, J. Rohrwild, "Baryon operators of higher twist in QCD and nucleon distribution amplitudes," Nucl. Phys. B 807 (2009) 89 [arXiv:0806.2531 [hep-ph]].

10. Y. Ji, A.V. Belitsky, "Renormalization of twist-four operators in light-cone gauge," arXiv:1405.2828 [hep-ph]. 